\title{
Therapeutic Potential of Sunitinib in Ameliorating Endothelial Dysfunction in Type 2 Diabetic Rats
}

\author{
Ali Mahdi ${ }^{a}$ Tong Jiao ${ }^{a}$ Yahor Tratsiakovich ${ }^{a}$ Bernhard Wernly ${ }^{a, b}, c, d$ \\ Jiangning Yang ${ }^{a}$ Claes-Göran Östenson ${ }^{\text {e }}$ A.H. Jan Danser ${ }^{f}$ John Pernow ${ }^{a}, g$ \\ Zhichao Zhou ${ }^{\text {a }}$ \\ aUnit of Cardiology, Department of Medicine, Karolinska Institutet, Stockholm, Sweden; ${ }^{b}$ Department of \\ Anaesthesiology, Perioperative Medicine and Intensive Care Medicine, Paracelsus Medical University of Salzburg, \\ Salzburg, Austria; 'Department of Cardiology, Paracelsus Medical University of Salzburg, Salzburg, Austria; \\ ${ }^{d}$ Center for Public Health and Healthcare Research, Paracelsus Medical University of Salzburg, Salzburg, Austria; \\ eDepartment of Molecular Medicine and Surgery, Endocrinology and Diabetology, Karolinska University Hospital, \\ Karolinska Institutet, Stockholm, Sweden; fDivision of Vascular Medicine and Pharmacology, Department of \\ Internal Medicine, Erasmus MC, University Medical Center Rotterdam, Rotterdam, The Netherlands; ${ }^{9}$ Department of \\ Cardiology, Karolinska University Hospital, Stockholm, Sweden
}

\section{Keywords}

Sunitinib · Endothelial function · Diabetes · Glucose ·

Microcirculation

\begin{abstract}
Introduction: Sunitinib, a multi-targeted tyrosine kinase receptor inhibitor used to treat renal-cell carcinoma and gastrointestinal stromal tumor, was recently shown to have a beneficial effect on metabolism in type 2 diabetes (T2D). Endothelial dysfunction is a key factor behind macro- and microvascular complications in T2D. The effect of sunitinib on endothelial function in T2D remains, however, unclear. We therefore tested the hypothesis that sunitinib ameliorates endothelial dysfunction in T2D. Methods: Sunitinib (2 mg/ kg/day, by gavage) was administered to T2D Goto-Kakizaki (GK) rats for 6 weeks, while water was given to GK and Wistar rats as controls. Hemodynamic, inflammatory, and metabolic parameters as well as endothelial function were measured. Results: Systolic, mean arterial blood pressures, plasma tu-
\end{abstract}

karger@karger.com www.karger.com/pha

Karger"

GOPEN ACCESS
(C) 2021 The Author(s).

Published by S. Karger AG, Basel

This is an Open Access article licensed under the Creative Commons Attribution-NonCommercial-4.0 International License (CC BY-NC) (http://www.karger.com/Services/OpenAccessLicense), applicable to the online version of the article only. Usage and distribution for commercial purposes requires written permission. mor necrosis factor a levels, kidney weight to body weight (BW) ratio, and glucose levels were higher, while BW was lower in GK rats than in Wistar rats. Six-week treatment with sunitinib in GK rats did not affect these parameters but suppressed the increase in glucose levels. Endothelium-dependent relaxations were reduced in both aortas and mesenteric arteries isolated from GK as compared to Wistar rats, which was markedly reversed in both types of arteries from GK rats treated with sunitinib. Conclusions: This study demonstrates that sunitinib has a glucose-lowering effect and ameliorates endothelial dysfunction in both conduit and resistance arteries of GK rats.

(c) 2021 The Author(s). Published by S. Karger AG, Basel

\section{Introduction}

Type 2 diabetes (T2D) is an important risk factor for the development of cardiovascular diseases, including atherosclerosis and ischemic heart disease [1]. Both mac- 
rovascular and microvascular complications significantly contribute to the increase in mortality and morbidity in the large group of patients with T2D [1]. Endothelial dysfunction represents an early hallmark for cardiovascular complications in patients with T2D and plays a pivotal role in the etiology of T2D-induced vascular complications. The underlying disease mechanisms for the development of endothelial dysfunction in T2D are complex, but important components are decreased bioavailability of nitric oxide and increased formation of reactive oxygen species $[2,3]$. The outcomes of clinical trials evaluating the effect of intensive glycemic control using established hypoglycemic agents, such as metformin and insulin, have not convincingly demonstrated overall beneficial effects on cardiovascular events [4]. New drug targets such as glucagon-like peptide- 1 receptor agonists and sodiumglucose cotransporter 2 inhibitor have been shown to exert beneficial effects on cardiovascular outcomes through glucose-independent mechanisms [5]. There is still a clinical need for improved understanding of the underlying disease mechanism in order to develop new therapeutic strategies for the treatment of vascular complications in T2D.

Sunitinib is an orally available tyrosine kinase inhibitor and the first-line therapy for treating metastatic renalcell carcinoma [6]. Sunitinib exerts its therapeutic effect via inhibition of several members of the split-kinase domain family of receptor tyrosine kinase including vascular endothelial growth factor receptor (VEGFR) 2 [6]. Despite side effects of hypertension and cardiac toxicity in cancer patients treated with sunitinib [7], emerging studies have shown a beneficial effect of sunitinib on overall metabolism in T2D [8]. Case reports have indicated that sunitinib treatment resulted in hypoglycemia in a nondiabetic patient [9] and improvement in glycemic control and need for less intensive glucose-lowering treatment in a patient with T2D [10]. Experimental studies demonstrated that sunitinib could prevent $\beta$-cell apoptosis and hemorrhage in the microcirculation of pancreatic islets, decrease glucose levels, and improve insulin sensitivity in spontaneously T2D rats [11]. Whether sunitinib also exerts beneficial effect on T2D-associated vascular complications remains unclear.

Consequently, we aimed to study the therapeutic potential of sunitinib for the treatment of endothelial dysfunction in T2D. Using Goto-Kakizaki (GK) rats, an established model of T2D that exhibits metabolic derangement and vascular dysfunction $[3,12,13]$, we evaluated the effect of chronic sunitinib treatment on endothelial function in both conduit and resistance arteries.

Sunitinib and Endothelial Dysfunction in Diabetes

\section{Materials and Methods}

\section{Drugs and Solutions}

Acetylcholine (ACh) and sodium nitroprusside (SNP) were purchased from Sigma-Aldrich (St. Louis, MO, USA). Sunitinib-L-malate (sunitinib) was obtained from patients who discontinued treatment at Erasmus University Medical Center, Rotterdam, The Netherlands [14]. All drugs were obtained with distilled water.

\section{Animals and Sunitinib Treatment}

All experimental protocols were performed in accordance with the Guide for Care and Use of Laboratory Animals (NIH publication No. 85-23, revised 1996) and approved by the regional Ethical Committee for animal experiments in Stockholm (17708-2019). Male Wistar rats were purchased from Charles River (Sulzfeld, Germany) and housed in the animal facility of Karolinska University Hospital (L5) and Karolinska Institutet (comparative medicine). GK rats were derived from glucose-intolerant Wistar rats and were bred in the animal facility. The GK strain was established from normoglycemic Wistar rats by repeated inbreeding in each successive generation of the siblings with the highest blood glucose levels during an oral glucose tolerance test [12]. All animals were kept at $22^{\circ} \mathrm{C}$, with 12-h light/ dark cycle and free access to standard chow and water. Wistar rats were treated with water and age- and sex-matched GK rats were treated with water or sunitinib ( $2 \mathrm{mg} / \mathrm{kg} /$ day, by gavage $)$ at the age of 10-15 weeks for 6 weeks. Body weight (BW), blood pressure (BP), and blood glucose were monitored before and at the end of the treatment. After the chronic treatment, animals were anesthetized with pentobarbital $(50 \mathrm{mg} / \mathrm{kg}$, i.p.) followed by collection of different organs and plasma. Wet kidney weight was measured, aortas and mesenteric arteries (MAs) were dissected for the evaluation of vascular function in the wire myograph, and insulin and tumor necrosis factor (TNF) a were measured (see below).

\section{Hemodynamic Measurement}

Rats were anesthetized with pentobarbital (50 mg/kg, i.p.). Right carotid artery was cannulated with a PE-50 catheter which was connected to a pressure transducer for $\mathrm{BP}$ registration. The animal was then tracheotomized and intubated to ventilate lungs with room air during the experiment. Arterial pressure was recorded with PharmLab V5.0 (AstraZeneca R\&D, Mölndal, Sweden).

\section{Immunoassay}

Plasma levels of TNFa and insulin were measured using ELISA (ThermoFisher Scientific, Waltham, MA, USA) according to the manufacturer's instructions.

\section{Tissue Preparation and Wire Myograph Study}

Rat thoracic aortas and MAs (the third-order branch) were cleaned by removing fat and connective tissues under microscope and subsequently cut transversely into $2-\mathrm{mm}$ rings. The vessels were then mounted on a wire myograph (Danish Myo Technology) in separate organ baths containing $6 \mathrm{~mL}$ Krebs-Henseleit (KH) buffer. The KH buffer ( $\mathrm{pH} 7.4$ ) containing (in $\mathrm{mM}$ ) $118 \mathrm{NaCl}, 4.7$ $\mathrm{KCl}, 1.2 \mathrm{MgSO}_{4}, 1.2 \mathrm{KH}_{2} \mathrm{PO}_{2}, 25 \mathrm{NaHCO}_{3}, 11$ glucose, and 2.4 $\mathrm{CaCl}_{2}$ was maintained at $37^{\circ} \mathrm{C}$ and aerated with $95 \% \mathrm{O}_{2} / 5 \% \mathrm{CO}_{2}$. 


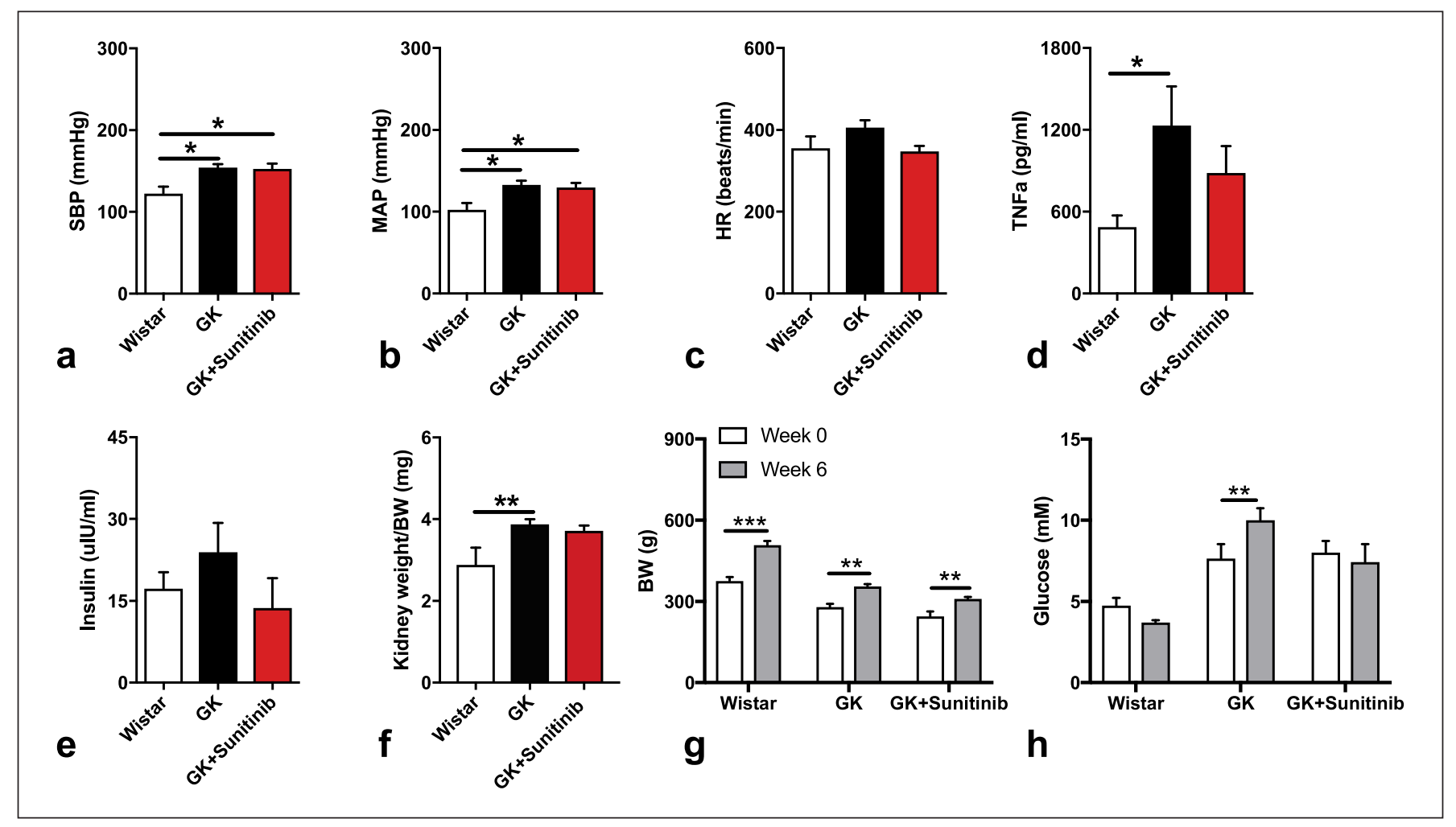

Fig. 1. Hemodynamic, inflammatory, and metabolic changes induced by sunitinib. Effect of sunitinib on $\operatorname{SBP}(\mathbf{a}, n=4)$, MAP (b, $n=4)$, HR (c, $n=4)$, plasma TNFa levels (d, $n=7-13)$, plasma insulin levels (e, $n=6-12)$, and kidney-to-BW ratio (f, $n=6-11)$. Measurement of BW (g) and blood glucose (h) at baseline and following 6-weeks sunitinib treatment in rats from 3 groups $(n=$

Changes in contractile forces were recorded with a Harvard isometric transducer. The vessel rings were exposed to $\mathrm{KCl}$ twice (50 $\mathrm{mM}$ and $100 \mathrm{mM}$, respectively for aortas; $50 \mathrm{mM}$ each time for MAs) to check the contractility. Thereafter, vessels were allowed to equilibrate in fresh $\mathrm{KH}$ buffer for $30 \mathrm{~min}$. Endothelium-dependent relaxation (EDR) was determined in phenylephrine (PE, 1 $\mu \mathrm{M})$-preconstricted vessels by administration of ACh $\left(10^{-9}-10^{-5}\right.$ $\mathrm{M})$. Endothelium-independent relaxation was evaluated with 10 $\mu \mathrm{M}$ SNP [3].

\section{Statistical Analysis}

Vascular relaxation to ACh or SNP was expressed as the percentage of contraction to PE. One-way ANOVA with post hoc Bonferroni's test or Kruskal-Wallis one-way ANOVA was used to compare multiple groups. A paired or unpaired $t$ test was used for comparison between 2 groups. Concentration responses were assessed using two-way ANOVA followed by post hoc Bonferroni's test. All data are represented as means \pm SEM. Two-sided $p<0.05$ was considered as statistically significant. Analyses were carried out with GraphPad Prism v.7 ( ).
4-10): Wistar rats, nontreated GK rats, and sunitinib-treated GK rats (GK + sunitinib). Week 0: treatment week 0 ; week 6: the end of treatment week 6. Data are presented as means \pm SEM. ${ }^{*} p<0.05$, ${ }^{* *} p<0.01,{ }^{* * *} p<0.001$. GK, Goto-Kakizaki; TNF, tumor necrosis factor; SBP, systolic blood pressure; MAP, mean arterial hypertension; $\mathrm{HR}$, heart rate; $\mathrm{BW}$, body weight.

\section{Results}

\section{Hemodynamics, Inflammatory, and Metabolic}

\section{Changes Induced by Sunitinib}

In accordance with previous studies $[15,16]$, the systolic BP and mean arterial pressure were significantly higher in GK than in Wistar rats (Fig. 1a, b). Sunitinib had no effect on systolic BP, mean arterial pressure, and heart rate (Fig. 1a-c). Plasma TNFa levels were significantly higher in GK rats versus Wistar rats, which was not affected by sunitinib treatment (Fig. 1d). Plasma insulin levels were not significantly different among Wistar, GK, and GK + sunitinib groups; despite that, there was a trend for higher levels in the GK versus Wistar group (Fig. 1e). The kidney-to-BW ratio was higher in GK rats versus Wistar rats but was not affected by sunitinib treatment in GK rats (Fig. 1f). In line with previous studies [3, 17], BW was lower in age-matched GK rats versus Wistar rats (g: 
Fig. 2. Effect of the 6-week treatment with sunitinib (2 $\mathrm{mg} / \mathrm{kg} /$ day) on endothelial function in T2D. EDR in response to increasing concentrations of $\mathrm{ACh}$ in aortic rings (a, $n=6-9)$ and MAs (b, $n=4-9$ ) preconstricted with $\mathrm{PE}$ isolated from Wistar, GK, and GK + sunitinib groups. Data are presented as means \pm SEM. ${ }^{* * *} p<0.001$ versus Wistar, ${ }^{\dagger} p<0.05,{ }^{\dagger \dagger \dagger} p<0.001$ versus GK. T2D, type 2 diabetes; ACh, acetylcholine; EDR, endothelium-dependent relaxation; PE, phenylephrine; MAs, mesenteric arteries; GK, Goto-Kakizaki.

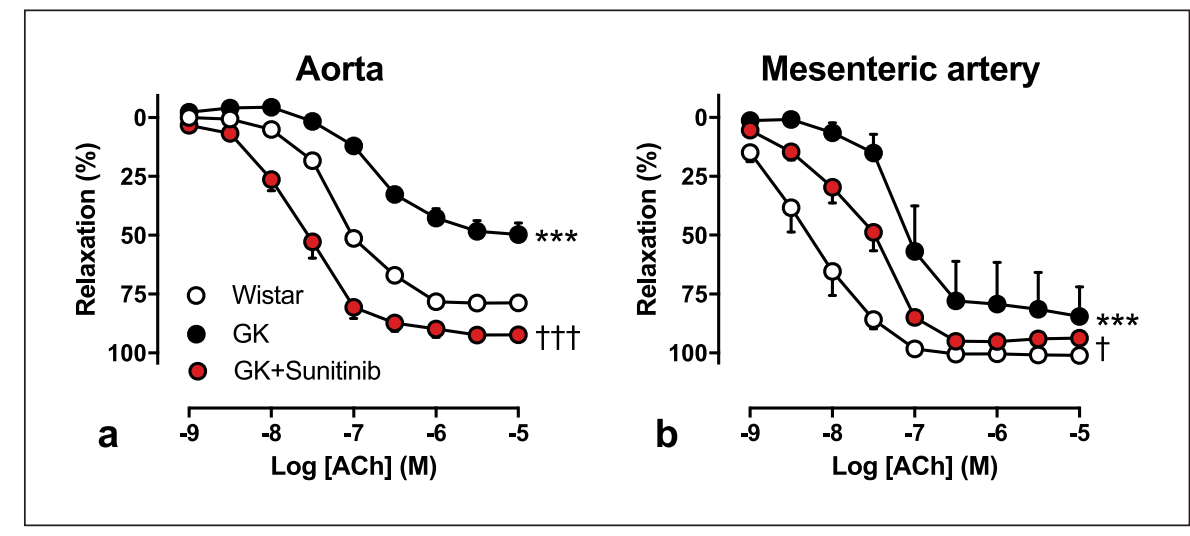

Table 1. $\mathrm{KCl}-$, $\mathrm{PE}-$, and $\mathrm{ACh}$-mediated vascular function in aortas and $\mathrm{MA}$

\begin{tabular}{llll}
\hline Vascular function, aorta/MA & Wistar & GK & GK + sunitinib \\
\hline $\mathrm{KCl}$ contraction $(\Delta \mathrm{mN})$ & $11.0 \pm 0.9 / 3.5 \pm 1.2$ & $9.9 \pm 1.0 / 5.7 \pm 1.2$ & $10.5 \pm 0.9 / 4.6 \pm 0.8$ \\
$\mathrm{PE}$ contraction $(\Delta \mathrm{mN})$ & $11.6 \pm 1.8 / 5.7 \pm 1.7$ & $10.2 \pm 1.5 / 10.1 \pm 2.2$ & $8.9 \pm 0.5 / 4.6 \pm 0.8$ \\
ACh relaxation $(-\operatorname{logEC} 50)$ & $7.2 \pm 0.05 / 8.3 \pm 0.12$ & $6.7 \pm 0.09 / 7.2 \pm 0.22$ & $7.7 \pm 0.07 / 7.6 \pm 0.08$ \\
\hline
\end{tabular}

Values are means $\pm \mathrm{SEM}$. No significant differences were detected for $\mathrm{KCl}$ and $\mathrm{PE}$ using Kruskal-Wallis one-way ANOVA. ACh, acetylcholine; MAs, mesenteric arteries; PE, phenylephrine; GK, Goto-Kakizaki.

$375 \pm 1.7$ in Wistar vs. $279 \pm 0.4$ in GK rats; $p<0.01$ ). The increases in BW during 6 weeks in GK rats were not affected by sunitinib treatment (Fig. 1g). Glucose levels were higher in GK rats versus Wistar rats at baseline (mM: $4.7 \pm 0.2$ in Wistar vs. $7.7 \pm 0.2$ in GK rats; $p<0.05$ ). Glucose levels were significantly increased over time in GK compared to Wistar rats (Fig. 1h). Of note, no increase in glucose levels was observed in GK rats treated with sunitinib (Fig. 1h). These observations indicate that sunitinib inhibited the increase in glucose levels over time but did not affect hemodynamics or inflammatory parameters in GK rats.

\section{Effect of Sunitinib Treatment on Endothelial Function in GK Rats}

The PE- and KCl-induced contraction in aortas and MAs did not differ significantly among Wistar, GK, and GK + sunitinib groups (Table 1). In accordance with previous studies $[3,18]$, endothelial dysfunction was present in GK rats as reflected by an impaired EDR in both aortas and MAs (Table 1; Fig. 2a, b). Following the 6-week sunitinib treatment in GK rats, EDR was markedly improved both in conduit aortas and in resistance MAs (Table 1; Fig. 2a, b). The SNP single concentration-induced endo- thelium-independent relaxation in these arteries was not affected by sunitinib (GK aorta: $97 \pm 1.3 \%$ vs. GK aorta+sunitinib: $99 \pm 0.2 \%$; GK mesenteric: $99 \pm 0.9 \%$ vs. GK mesenteric+sunitinib: $98 \pm 1.8 \%)$. These findings indicate that chronic treatment with sunitinib ameliorates endothelial dysfunction in both conduit and resistance arteries in GK rats.

\section{Discussion}

To the best of our knowledge, this is the first study evaluating the potential therapeutic effect of sunitinib on endothelial function as an endpoint in T2D. The main findings are that chronic treatment with sunitinib had glucose-lowering effect in GK rats and markedly ameliorated endothelial dysfunction in both conduit aortas and resistance MAs isolated from GK rats (Fig. 3). These results suggest that sunitinib exerts beneficial vascular and glucometabolic effects in T2D with potentially important implications which are discussed below.

Sunitinib is the first-line strategy for treating metastatic renal-cell carcinoma via inhibition of receptor tyrosine kinase including VEGFR2 [6]. Several lines of evidence 


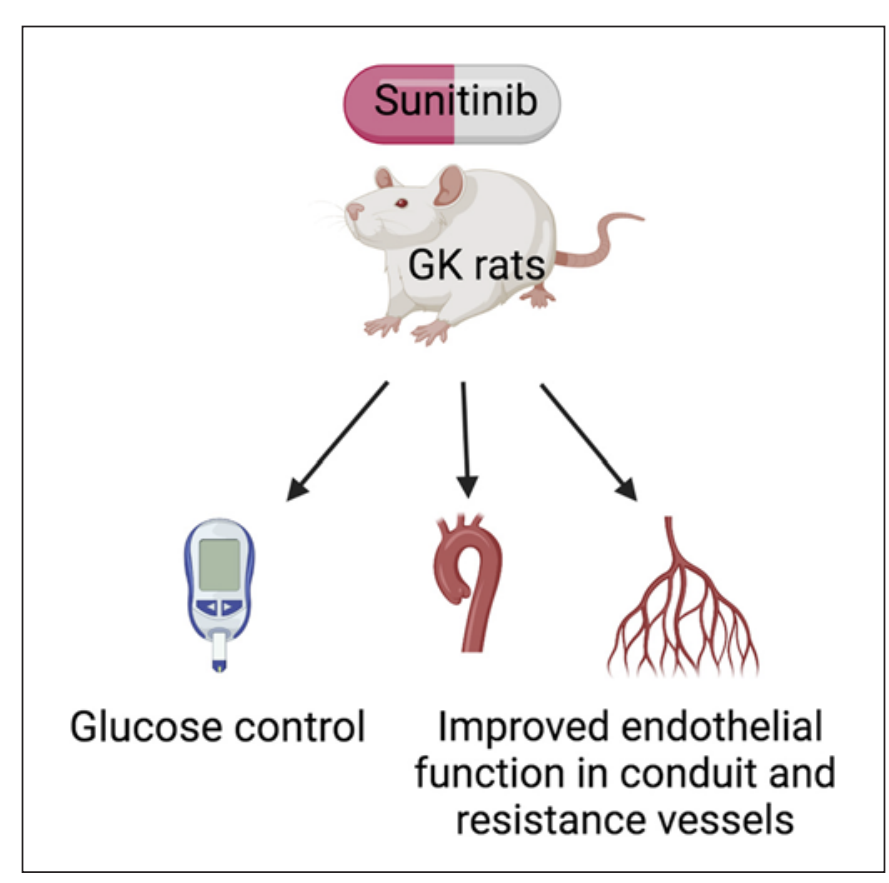

Fig. 3. Schematic summary of the study. Chronic treatment with sunitinib in type 2 diabetic GK rats significantly improves the glucose control and attenuates endothelial dysfunction in both conduit aortas and resistant MAs. GK, Goto-Kakizaki; MAs, mesenteric arteries (generated with BioRender under license).

suggest that sunitinib may have favorable effects on the diabetic metabolism. In a case report, it was described that a patient with $\mathrm{T} 2 \mathrm{D}$ experienced improvement in glycemic control and less intensive anti-glycemic treatment following sunitinib treatment [10]. Sunitinib prevented $\beta$-cell apoptosis and hemorrhage in the microcirculation of pancreatic islets, decreased glucose levels, and improved insulin sensitivity in spontaneously T2D rats [11]. Of interest, a single intravitreous injection of sunitinib microparticles in an animal model was recently shown to suppress choroidal neovascularization for 6 months and to block VEGFR-mediated leukostasis and retinal nonperfusion, which are associated with diabetic retinopathy progression [19]. This suggests that sunitinib has potential to be beneficial in the treatment of diabetes-induced vascular complications. Based on the fact that endothelial dysfunction plays a pivotal role in the etiology of T2Dinduced vascular complications, we set out to investigate the therapeutic potential of sunitinib in attenuating endothelial dysfunction in T2D. We found that chronic treatment with sunitinib not only had glucose-lowering effect in GK rats but also attenuated endothelial dysfunction in both conduit aortas and resistance MAs. These observations support the concept that sunitinib has the potential to be beneficial in the treatment of diabetes-induced vascular complications. The beneficial effect of sunitinib on endothelial function in GK rats may be associated with the glucose-lowering effect, which can lead to increase in nitric oxide bioavailability and decrease in reactive oxygen species formation [20,21]. It is also likely that the improvement is in part attributed to the direct effect of sunitinib on the endothelium as sunitinib treatment or inhibition of the VEGFA-VEGFR2 pathway prevented aneurysm formation and progression in mice [22]. However, such benefits are unlikely to be associated with attenuation of inflammatory and insulin signaling pathways as sunitinib had no significant effects on inflammatory marker TNFa and insulin levels. The exact mechanisms underlying attenuation of endothelial dysfunction by sunitinib are not readily known and warrant further investigations.

It is important to note that treatment with sunitinib can induce hypertension in both clinical and experimental studies, which is known as a common side effect of cancer treatment [14, 23-26]. The mechanistic insights underlying the rise in BP in both humans and animals have pointed to the involvement of endothelin $[14,23$, 27]. This seems to rely on different dosages of sunitinib applied [8]. The dose of sunitinib $(2 \mathrm{mg} / \mathrm{kg})$ used in the present study is comparable to those $(1.5-2.5 \mathrm{mg} / \mathrm{kg})$ used in experimental studies reporting the beneficial effect for diabetes, although the side effects were not checked but is lower than those used in studies demonstrating hypertensive effect and upregulation of the endothelin system $(7-26.7 \mathrm{mg} / \mathrm{kg})[11,25]$. In the present study, sunitinib treatment did not further increase BP of GK rats whose BP was already high as compared to Wistar rats at the basal condition. This is also reflected by the lack of effect on the heart rate in GK rats following sunitinib treatment. The already high BP at baseline in GK rats may mask the side effect by sunitinib. It should also be noted that the sunitinib-induced hypertension may also affect endothelial function depending on the dose of sunitinib. Thus, endothelial dysfunction was observed in rats treated with a high dose of sunitinib $(26.7 \mathrm{mg} / \mathrm{kg})$ [14], while endothelial function was preserved in rats with $14 \mathrm{mg} / \mathrm{kg}$ sunitinib [28]. This should be compared to the dose of $2 \mathrm{mg} / \mathrm{kg}$ in the present study resulting in improved endothelial function in T2D. Thus, a low dose seems to exert beneficial vascular effects with no negative side effect on BP.

Some limitations are worth mentioning. First, endothelial function was evaluated ex vivo, which does not ful- 
ly reflect the in vivo situation. Sunitinib may exert effects on multiple systems in vivo to influence endothelial function in T2D. The effect of sunitinib on other vascular aspects, for example, vascular remodeling warrants further investigations. Moreover, the dose of sunitinib $(2 \mathrm{mg} / \mathrm{kg})$ used in the present study is similar to those used in experimental studies reporting the beneficial effect for diabetes, although side effects were not checked (1.5-2.5 mg/ $\mathrm{kg}$ ) but is lower than those used in studies demonstrating a hypertensive effect and upregulation of the endothelin system $(7-26.7 \mathrm{mg} / \mathrm{kg})[11,25]$. However, this dosage was only applied to GK rats. The optimal dose of sunitinib needs to be tested in both T2D and healthy groups to control for the side effect and to achieve the maximal therapeutic effect for the favorable vascular effects in T2D.

In conclusion, the present study demonstrates that chronic treatment with sunitinib improves glucose control and ameliorates endothelial dysfunction in both conduit and resistance arteries of T2D rats without major cardiovascular side effects. This may pave the way for additional endpoints in the future trials, evaluating the clinical efficacy of sunitinib.

\section{Acknowledgment}

We thank Marita Wallin for excellent technical assistance.

\section{Statement of Ethics}

This study was conducted in accordance with the Guide for Care and Use of Laboratory Animals (NIH publication No. 85-23, revised 1996) and approved by the regional Ethical Committee for animal experiments in Stockholm (17708-2019).

\section{Conflict of Interest Statement}

The authors have no conflicts of interest to declare.

\section{Funding Sources}

This study was supported by the Swedish Heart and Lung Foundation (20190341 and 20200326 to Z.Z. and 20190266 to J.P.), the Loo and Hans Ostermans Foundation (2018-01213 and 202001209 to Z.Z.), the Karolinska Institute Grant (2018-01837 and 2020-01473) (to Z.Z.), the Lars Hiertas Minne Foundation (FO2018-0156 to Z.Z.), the Eva and Oscar Ahréns Foundation (2021-1233 to Z.Z.), the Swedish Research Council (2020-01372 to J.P.), the Diabetes Research Wellness Foundation (720-1519-16 and 363-PG to J.P.), the Stockholm County Council ALF (20190031 to J.P.), and the EFSD/Sanofi European Diabetes Research Programme in Macrovascular Complications (to J.P.).

\section{Author Contributions}

Z.Z. conceived and designed the study; A.M., T.J., Y.T., B.W., and Z.Z. performed and collected research data; A.M., T.J., and Z.Z. analyzed research data and performed statistical analyses; A.M., T.J., Y.T., B.W., J.Y., C.G.O., A.H.D., J.P., and Z.Z. contributed to discussion; A.M. and Z.Z. wrote the manuscript; A.M., T.J., J.P., and Z.Z. edited the manuscript; and all the authors reviewed the final version of the manuscript.

\section{Data Availability Statement}

All data generated or analyzed are included in this article. Further inquiries can be directed to the corresponding author.

\section{References}

1 Paneni F, Beckman JA, Creager MA, Cosentino F. Diabetes and vascular disease: pathophysiology, clinical consequences, and medical therapy: part I. Eur Heart J. 2013 Aug; 34(31):2436-43.

2 Gimbrone MA Jr, García-Cardeña G. Endothelial cell dysfunction and the pathobiology of atherosclerosis. Circ Res. 2016 Feb 19; 118(4):620-36.

3 Mahdi A, Jiao T, Tratsiakovich Y, Yang J, Ostenson CG, Pernow J, et al. Altered purinergic receptor sensitivity in type 2 diabetes-associated endothelial dysfunction and $\mathrm{Up}(4) \mathrm{A}$ mediated vascular contraction. Int J Mol Sci. 2018 Dec 7;19(12):3942.

Sunitinib and Endothelial Dysfunction in Diabetes
4 Palmer SC, Mavridis D, Nicolucci A, Johnson DW, Tonelli M, Craig JC, et al. Comparison of clinical outcomes and adverse events associated with glucose-lowering drugs in patients with type 2 diabetes: a meta-analysis. JAMA. 2016 Jul 19;316(3):313-24.

5 Lee MMY, Petrie MC, McMurray JJV, Sattar N. How do SGLT2 (Sodium-Glucose Cotransporter 2) inhibitors and GLP-1 (Glucagon-Like Peptide-1) receptor agonists reduce cardiovascular outcomes? Completed and ongoing mechanistic trials. Arterioscler Thromb Vasc Biol. 2020 Mar;40(3):506-22.

6 Rizzo M, Porta C. Sunitinib in the treatment of renal cell carcinoma: an update on recent evidence. Ther Adv Urol. 2017 Aug;9(8):195207.
7 Yang Y, Bu P. Progress on the cardiotoxicity of sunitinib: prognostic significance, mechanism and protective therapies. Chem Biol Interact. 2016 Sep 25;257:125-31.

8 Mahdi A, Wernly B, Pernow J, Zhou Z. Sunitinib and its effect in the cardiovascular system. Drug Discov Today. 2021;26:1773-5.

9 Demirci A, Bal O, Durnali A, Ekinci AS, Esbah O, Alkis N, et al. Sunitinib-induced severe hypoglycemia in a diabetic patient. J Oncol Pharm Pract. 2014 Dec;20(6):469-72.

10 Tyrrell HE, Pwint T. Sunitinib and improved diabetes control. BMJ Case Rep. 2014 Dec 24; 2014:bcr2014207521. 
11 Mukai E, Ohta T, Kawamura H, Lee EY, Morita A, Sasase T, et al. Enhanced vascular endothelial growth factor signaling in islets contributes to beta cell injury and consequential diabetes in spontaneously diabetic Torii rats. Diabetes Res Clin Pract. 2014 Nov; 106(2):303-11.

12 Goto Y, Kakizaki M, Masaki N. Production of spontaneous diabetic rats by repetition of selective breeding. Tohoku J Exp Med. 1976 May;119(1):85-90.

13 Kobayashi T, Matsumoto T, Ooishi K, Kamata K. Differential expression of alpha2D-adrenoceptor and eNOS in aortas from early and later stages of diabetes in Goto-Kakizaki rats. Am J Physiol Heart Circ Physiol. 2004 Jul; 287(1):H135-43.

14 Kappers MH, van Esch JH, Sluiter W, Sleijfer S, Danser AH, van den Meiracker AH. Hypertension induced by the tyrosine kinase inhibitor sunitinib is associated with increased circulating endothelin-1 levels. Hypertension. 2010 Oct;56(4):675-81.

15 Cui W, Zheng Y, Zhang Q, Wang J, Wang L, Yang W, et al. Low-molecular-weight fucoidan protects endothelial function and ameliorates basal hypertension in diabetic GotoKakizaki rats. Lab Invest. 2014 Apr;94(4): 382-93.

16 Alameddine A, Fajloun Z, Bourreau J, Gauquelin-Koch G, Yuan M, Gauguier D, et al. The cardiovascular effects of salidroside in the
Goto-Kakizaki diabetic rat model. J Physiol Pharmacol. 2015 Apr;66(2):249-57.

17 Szkudelska K, Deniziak M, Hertig I, Wojciechowicz T, Tyczewska M, Jaroszewska $\mathrm{M}$, et al. Effects of resveratrol in Goto-Kakizaki rat, a model of type 2 diabetes. Nutrients. 2019 Oct 16;11(10):2488.

18 Sena CM, Matafome P, Louro T, Nunes E, Fernandes R, Seiça RM. Metformin restores endothelial function in aorta of diabetic rats. Br J Pharmacol. 2011 May;163(2):424-37.

19 Tsujinaka H, Fu J, Shen J, Yu Y, Hafiz Z, Kays $J$, et al. Sustained treatment of retinal vascular diseases with self-aggregating sunitinib microparticles. Nat Commun. 2020 Feb 4;11(1): 694.

20 Wright E Jr, Scism-Bacon JL, Glass LC. Oxidative stress in type 2 diabetes: the role of fasting and postprandial glycaemia. Int J Clin Pract. 2006 Mar;60(3):308-14.

21 Shi Y, Vanhoutte PM. Macro- and microvascular endothelial dysfunction in diabetes. J Diabetes. 2017 May;9(5):434-49.

22 Xu B, Iida Y, Glover KJ, Ge Y, Wang Y, Xuan $\mathrm{H}$, et al. Inhibition of VEGF (Vascular Endothelial Growth Factor)-A or its receptor activity suppresses experimental aneurysm progression in the aortic elastase infusion model. Arterioscler Thromb Vasc Biol. 2019 Aug; 39(8):1652-66.

23 Kappers MH, de Beer VJ, Zhou Z, Danser AH, Sleijfer S, Duncker DJ, et al. Sunitinib-in- duced systemic vasoconstriction in swine is endothelin mediated and does not involve nitric oxide or oxidative stress. Hypertension. 2012 Jan;59(1):151-7.

24 Hamnvik OP, Choueiri TK, Turchin A, McKay RR, Goyal L, Davis M, et al. Clinical risk factors for the development of hypertension in patients treated with inhibitors of the VEGF signaling pathway. Cancer. 2015 Jan 15;121(2):311-9.

25 Lankhorst S, Baelde HJ, Kappers MH, Smedts FM, Hansen A, Clahsen-van Groningen MC, et al. Greater sensitivity of blood pressure than renal toxicity to tyrosine kinase receptor inhibition with sunitinib. Hypertension. 2015 Sep;66(3):543-9.

26 Ravaud A, Motzer RJ, Pandha HS, George DJ, Pantuck AJ, Patel A, et al. Adjuvant sunitinib in high-risk renal-cell carcinoma after nephrectomy. N Engl J Med. 2016 Dec 8;375(23): 2246-54.

27 Neves KB, Touyz RM. Extracellular vesicles as biomarkers and biovectors in primary aldosteronism. Hypertension. 2019 Aug;74(2): 250-2.

28 Mirabito Colafella KM, Neves KB, Montezano AC, Garrelds IM, van Veghel R, de Vries $\mathrm{R}$, et al. Selective ETA vs. dual ETA/B receptor blockade for the prevention of sunitinib-induced hypertension and albuminuria in WKY rats. Cardiovasc Res. 2020 Aug 1;116(10): 1779-90. 